\title{
Acute, isolated and unstable syndesmotic injuries are frequently associated with intra-articular pathologies
}

\author{
Kathrin Rellensmann ${ }^{1}$. Cyrus Behzadi ${ }^{2}$ John Usseglio ${ }^{3}$. James Turner Vosseller ${ }^{4}$. Wolfgang Böcker ${ }^{1}$. \\ Hans Polzer ${ }^{1,4}$. Sebastian Felix Baumbach ${ }^{1}$
}

Received: 6 March 2020 / Accepted: 2 July 2020 / Published online: 29 July 2020

(c) The Author(s) 2020

\begin{abstract}
Purpose Although simultaneous arthroscopy for the surgical treatment of acute isolated, unstable syndesmotic injuries has been recommended, little knowledge is present about the actual frequency of intra-articular pathologies for this injury. The aim of this study was to investigate the frequency and severity of intra-articular pathologies detected during arthroscopy and their subsequent treatment in acute isolated, unstable syndesmotic injuries.

Methods A retrospective chart review of patients treated by arthroscopic-assisted stabilization for acute isolated, syndesmotic instability was performed. The primary outcome parameter was the frequency of intra-articular pathologies. Secondary outcome parameters were the type of syndesmotic lesion (ligamentous/bony), severity of chondral lesions, MRI findings, treatment details, complications and the identification of factors associated with intra-articular pathologies.

Results Twenty-seven patients, $19 \%$ female, with a mean age of $37 \pm 12$ years met the inclusion criteria. $70 \%$ suffered isolated ligamentous injuries, the remaining suffered avulsion fractures of the syndesmosis. Chondral lesions occurred in $48 \%$ (ICRS grade II: 33\%; ICRS grade IV 15\%) and intra-articular loose bodies in 11\% of patients. Overall, arthroscopy revealed intra-articular pathologies necessitating further treatment in 19\% of patients. Neither the type of syndesmotic injury (bony vs. ligamentous; ns) nor the degree of ligamentous instability (West Point IIB vs. III; ns) had a significant influence on the occurrence of chondral lesions. One complication (SSI) occurred. Pre-operative MRI revealed a sensitivity/specificity of $100 / 79 \%$ for chondral lesions and 50/93\% for loose bodies.

Conclusion Intra-articular pathologies in acute isolated, unstable syndesmotic injuries occur in up to 50\% of patients, $19 \%$ necessitated additional treatment. Simultaneous arthroscopy, independent of the pre-operative MRI findings, appears reasonable in highly active patients.
\end{abstract}

Level of evidence Level III.

Keywords Syndesmotic injury $\cdot$ Syndesmosis $\cdot$ High ankle sprains $\cdot$ Arthroscopy $\cdot$ Chondral lesions $\cdot$ Surgery

Electronic supplementary material The online version of this article (https://doi.org/10.1007/s00167-020-06141-y) contains supplementary material, which is available to authorized users.

Hans Polzer

hans.polzer@med.uni-meunchen.de

1 Department of General, Trauma and Reconstructive Surgery, University Hospital, LMU Munich, Nussbaumstraße 20, 80336 Munich, Germany

2 Radiologie München, Dienerstraße 12, 80331 Munich, Germany

3 Long Health Sciences Library, Columbia University Irving Medical Center, New York, USA

4 Department of Orthopaedic Surgery, Columbia University Medical Center, New York, NY, USA

\author{
Abbreviations \\ CT Computed tomography \\ ICRS International Cartilage Repair Society \\ MRI Magnetic resonance imaging \\ SSI Surgical side infection \\ ns Not significant
}

\section{Introduction}

Isolated lesions of the syndesmosis, i.e. "high ankle sprains", contribute only $1-17 \%$ of all ankle sprains in the general population $[19,21]$. Yet, in high impact sports, they make up for up to $30 \%$ of all ankle sprains [25]. Multiple different classification systems for isolated syndesmotic injuries 
have been published [37], most of which differentiate acute (3-4 weeks), from subacute (to 12 weeks) and chronic injuries [13, 27], as well as stable and unstable injuries. The stability of syndesmotic injuries most often is assessed by the West Point Ankle Grading System [19, 37] in its adaptation from Calder et al. [6]. Most authors agree that grade IIB and III lesions necessitate surgical stabilization [6]. Today, surgical treatment consists of closed reduction of the distal tibiofibular joint and indirect fixation, either static or dynamic. Although static fixation by means of one or two syndesmotic screws is considered to be the most common procedure, emerging evidence points at a superior patient reported outcome for the dynamic fixation by a suture button system [31].

It is worth noting that accompanying intra-articular pathologies, such as loose bodies and chondral lesions, have been reported in up to $30 \%$ of patients suffering chronic lateral ankle instability [7, 15, 22] and in up to $80 \%$ of patients with ankle fractures $[4,20]$. To identify and treat these pathologies, additional arthroscopy is recommended during the surgical treatment of both, chronic ankle instability [23, 39] and complex ankle fractures [34, 35]. Magnetic resonance imaging (MRI) is often considered a non-invasive alternative to pre-operatively identify patients with intraarticular pathologies. Still, MRI has been reported to have a limited sensitivity in detecting chondral lesion and loose bodies $[2,18,26,30]$. Arthroscopy on the other hand does not only allow to reliably identify and treat intra-articular pathologies but also seems to improve the patient reported outcomes [23, 34, 35, 39]. Considering that syndesmotic injuries result from a comparable injury mechanism, i.e. ankle sprains, it can be suspected that intra-articular pathologies also occur frequently in patients suffering an unstable syndesmotic injury. Particularly because syndesmotic injuries occur predominantly in a physically highly active population, it seems reasonable that these patients would benefit from arthroscopic-assisted surgery.

In the course of this study, we conducted a thorough literature review to identify studies reporting on intra-articular pathologies in patients treated for an acute isolated, unstable syndesmotic injury. Although various authors have recommended arthroscopy $[11,14,38]$, only one study mentioned chondral lesions detected by arthroscopy. Unfortunately, these were only reported as a side note, and neither their severity nor their treatment was documented [8]. Consequently, no study has yet in detail assessed the frequency of intra-articular pathologies in patients suffering an acute isolated, unstable syndesmotic injury.

The authors hypothesized that intra-articular lesions are common in patients suffering an acute isolated, unstable syndesmotic injury. Up to date, the frequency of accompanying intra-articular lesions in acute isolated, unstable syndesmotic injury is unknown. Therefore, the purpose of this study was to investigate the frequency and severity of intra-articular pathologies detected during arthroscopy and their subsequent treatment in acute isolated, unstable syndesmotic injuries.

\section{Materials and methods}

After receiving approval from the ethics committee of the University Hospital, LMU Munich (IRB ID: 19-868), a retrospective chart review was performed to identify patients treated by arthroscopic-assisted stabilization of acute syndesmotic instability.

\section{Patient cohort}

A retrospective chart review was conducted based on the ICD-10: S93.2 as well as OPS-2019: 5-869.2 and 1-697.8 between 01/2014 and 09/2019. Inclusion criteria were patient age $\geq 18$ years and arthroscopic-assisted surgical treatment of acute isolated, unstable syndesmotic injuries. An injury was considered acute if it was treated within the first 12 weeks after trauma [28, 32, 36]. Isolated syndesmotic injuries were defined as ligamentous injuries and/or syndesmotic avulsion fractures, i.e. fracture to the posterior malleolus, Tubercule de Tillaux Chaput or Wagstaff fragment [3]. Unstable was defined as a two-ligament lesion with instability proven during arthroscopy or functional radiographic examination. Syndesmotic instability, at our department, is assessed using radiograph, MRI, CT and, in case of uncertainty, an "external rotation test" under fluoroscopy [17] is performed. Instability was defined as either dislocated bony avulsion of the syndesmotic complex (posterior malleolus, Tubercule de Tillaux Chaput, or Wagstaff fragment) or positive external rotation test under fluoroscopy in pure ligamentous injuries.

\section{Treatment}

Our Foot and Ankle Division is part of the Department of General, Trauma and Reconstructive Surgery. Depending on the paths of admission, patients are either seen by the trauma surgeon on call or referred to our outpatient clinic. Starting in mid-2014, patients suffering an acute isolated, unstable syndesmotic injury presenting to the Division of Foot and Ankle Division were routinely treated by additional arthroscopy. Arthroscopy was first performed utilizing the standard anteromedial and -lateral portals. No traction device was applied. Impinging soft tissue and loose bodies were removed. Then the joint was explored, and cartilage lesions graded according to the recommendations of the International Cartilage Repair Society (ICRS; [5]). In case of ICRS grade II and III lesions, chondroplasty was performed per the 
choice of the surgeon. ICRS grade IV lesions were all treated by micro-/nanofracturing. Non-dislocated PM fragments of sufficient size were fixed percutaneously by AP screws and dislocated ventral bony syndesmotic avulsion (Tubercule de Tillaux Chaput, or Wagstaff fragment) by open reduction and internal fixation. The distal tibiofibular joint was stabilized by suture button(s) (TightRope, Fa. Arthrex, Naples, FL, USA) \pm a syndesmotic screw or internal brace, per the surgeon's preference. Patients admitted to our Trauma Division but not to the Division of Foot and Ankle Surgery, were all treated by a fellowship trained trauma surgeon. They utilized the same protocol to treat bony and ligamentous syndesmotic injuries. However, no additional arthroscopy was performed and, therefore, these patients were excluded.

\section{Primary and secondary outcome parameters}

The primary outcome parameter was the incidence of intraarticular pathologies, i.e. chondral lesions, loose bodies or osteochondral lesions, detected during arthroscopy. Secondary outcome parameters were the severity of the cartilage lesions according to the ICRS grading system [5], its localization per the anatomical grid scheme [12], treatment details including treatment of the cartilage lesion, fixation of avulsion fractures, type of syndesmotic stabilization and complications related to the additional arthroscopy. In addition, all available preoperative MRI scans were anonymized and reviewed by a blinded senior radiologist with special interest in musculoskeletal and cartilage imaging. In concordance with the arthroscopic grading, the severity and localization of the cartilage lesions were rated per the ICRS grading system [5] and the anatomical grid scheme [12], respectively. Any intra-articular loose body was documented. For image analysis, a standard post-processing workstation was used in all data sets. The blinded radiological findings were then compared to the intra-operative findings.

\section{Statistical analysis}

Standard descriptive statistics, independent t test, and Chi square tests were performed using SPSS (Version 25, IBM, Armonk, United States). If not stated differently, values are given a mean \pm SD. The level of significance was set to $p<0.05$.

\section{Results}

The database search yielded 697 results. Out of these, 53 patients suffered an acute, isolated, unstable syndesmotic injury. Twenty-seven of which were treated with additional arthroscopy (Fig. 1). The demographic details including the ASA score, trauma mechanism and injury type are outlined

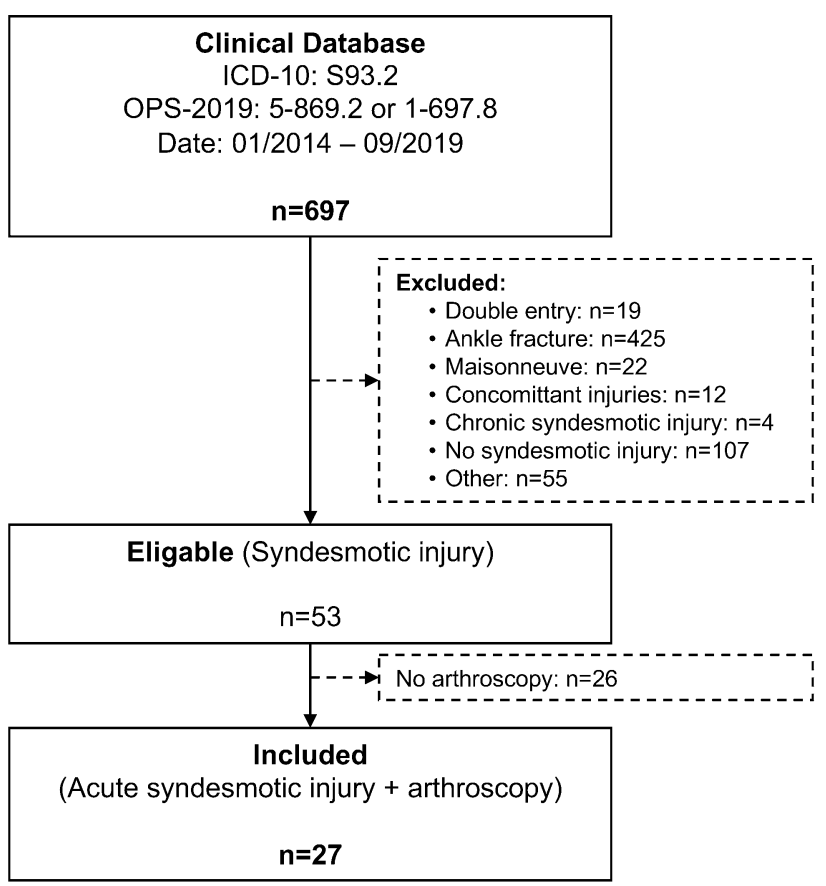

Fig. 1 Flow-chart illustrating the patient selection process. ICD10 S93.2: Traumatic rupture of ligaments of the ankle or foot; OPS 5-869.2: Stabilization of syndesmotic lesions with fixation methods; OPS 1-697.8: Diagnostic arthroscopy of the upper ankle joint; Concomitant injuries: Fractures of the tibial shaft or metatarsal fractures; Other: Ligamentous injury of the elbow or knee

in Table 1, as well as a comparison to the excluded nonarthroscopic cohort. Both cohorts showed no significant differences, but significantly more bony avulsion syndesmotic lesion were found in the arthroscopy cohort $(p=0.024)$.

The detailed injury patterns and treatment details per patient are shown in supplement 1 . Per the primary outcome parameter, 15 chondral lesions occurred in 13 (48\%) patients. In four patients, there occurred five (15\%) ICRS grade IV lesions, which necessitated treatment by micro-/ nanofracturing. No osteochondral lesions were observed. $76 \%$ of cartilage lesion were talar (Fig. 2), $24 \%$ tibial.

Loose bodies were detected and removed in $3(11 \%)$ patients, two of which also had an ICRS grade IV chondral lesion. Neither the type of syndesmotic injury (bony vs. ligamentous; ns) nor the degree of ligamentous instability (West Point IIB vs. III; ns) had a significant influence on the occurrence of chondral lesions. ICRS grade IV lesions occurred in 2 out of $18(11 \%)$ patients with an isolated ligamentous syndesmotic injury and in 2 out of $9(22 \%)$ patients with a bony avulsion (ns). Overall, five (19\%) patients presented with an intra-articular lesion (four patients with chondral injuries grade IV, one patient with loose bodies) necessitating additional treatment. One complication (4\%), i.e. surgical site infection (SSI) with revision surgery of the anteriorlateral portal, was observed in the herein presented cohort. 
Table 1 Comparing the included arthroscopy cohort to the excluded non-arthroscopy cohort

\begin{tabular}{|c|c|c|c|}
\hline & Arthroscopy cohort $(n=27)$ & $\begin{array}{l}\text { Non-arthroscopy } \\
\text { cohort—excluded } \\
(n=26)\end{array}$ & $p$ value \\
\hline Age (years) & $37 \pm 12$ & $34 \pm 16$ & ns \\
\hline$\%$ Female & $19 \%$ & $41 \%$ & ns \\
\hline ASA score & $1.9 \pm 0.6$ & $1.6 \pm 0.7$ & ns \\
\hline \multicolumn{4}{|l|}{ Trauma mechanism } \\
\hline Ankle sprain & $82 \%$ & $90 \%$ & ns \\
\hline Fall from height $<1 \mathrm{~m}$ & $14 \%$ & $5 \%$ & \\
\hline High energy trauma & $4 \%$ & $5 \%$ & \\
\hline Isolated ligamentous syndesmotic injury & $\begin{array}{l}\text { 67\% (Westpoint IIB: 48\%; } \\
\text { Westpoint III: 19\%) }\end{array}$ & $\begin{array}{l}\text { 95\% (Westpoint } \\
\text { IIB: } 81 \% \text {; West- } \\
\text { point III: } 38 \% \text { ) }\end{array}$ & 0.024 \\
\hline Bony avulsion syndesmotic lesions & $33 \%$ & $5 \%$ & \\
\hline
\end{tabular}

ASA, American Society of Anesthesiologists Classification; m, meter; n, number

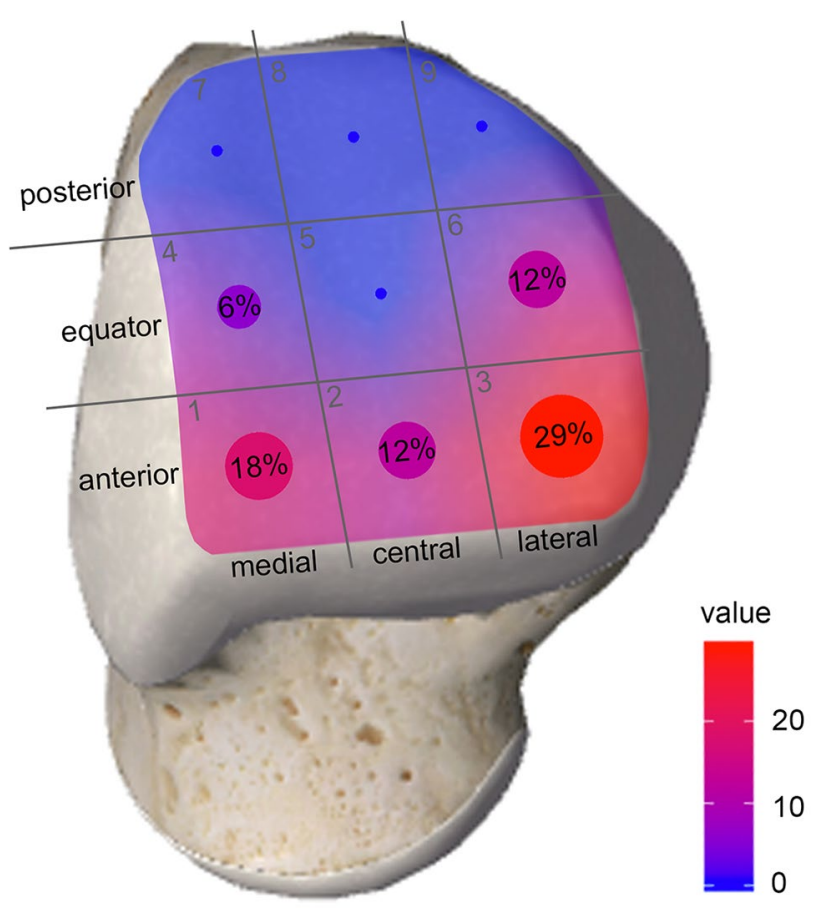

Fig. 2 Illustration of the localization of the talar chondral lesions per the anatomical grid scheme

Pre-operative MRIs were available for review in 17 patients (74\%). As most of the MRI images had not been conducted at our hospital, their quality varied considerable. Overall, MRIs were generated on 9 different scanners, $71 \%$ of which were 1.5 Tesla, the remaining 3 Tesla devices. Based on a four-item Likert scale (1 very good, 4 poor), the MRI image quality was overall rated as good $(2.0 \pm 0.9)$. The MRI review resulted in six chondral lesions ICRS grade IV (35\% of patients), two lesions ICRS grade I to III (12\% of patients), and two loose bodies (12\% patients). Overall, in $35 \%$ of patients, arthroscopy would have been indicated
Table 2 Four-fold table of MRI findings on chondral lesions and loose bodies compared to the arthroscopic findings

\begin{tabular}{llccc}
\hline & ICRS IV & ICRS $\leq$ III & Loose bodies & $\begin{array}{l}\text { No } \\
\text { loose } \\
\text { bodies }\end{array}$ \\
\hline Positive & 3 & 3 & 1 & 1 \\
Negative & 0 & 11 & 1 & 14 \\
\hline
\end{tabular}

based on the MRI findings (chondral lesion ICRS grade IV \pm loose bodies). Based on the four-fold table in Table 2, MRI revealed a sensitivity/specificity of $100 / 79 \%$ for chondral lesions and 50/93\% for loose bodies. The positive predictive/negative predictive value for chondral lesions were $0.5 / 1$ and $0.5 / 0.93$ for loose bodies.

\section{Discussion}

The aim of this study was to investigate the frequency and severity of intra-articular pathologies detected during arthroscopy and their subsequent treatment in acute isolated, unstable syndesmotic injuries. Twenty-seven patients were retrospectively reviewed and arthroscopy revealed chondral lesions in $48 \%$ and loose bodies in $11 \%$. Overall, $19 \%$ of patients presented with intra-articular pathologies necessitated additional treatment, i.e. micro-/nanofracturing and/ or removal of loose bodies. On the contrary, pre-operative MRI revealed chondral lesions in 35\% and loose bodies in $12 \%$ of the patients. Based on the pre-operative MRI findings, additional arthroscopy would have been indicated in $35 \%$ of the patients.

As stated in the introduction, we initially conducted a thorough literature review. Only one study on arthroscopically detected intra-articular pathologies during surgical treatment of acute isolated, unstable syndesmotic 
injuries was identified. D'Hooghe et al. [8] presented a retrospective study on 110 male professional football players who underwent arthroscopic assisted treatment for acute ( $<6$ weeks), isolated unstable (West Point grade $\geq$ IIB) syndesmosis injuries. Their primary outcome was sport-specific rehabilitation, but cartilage injuries were also noted, which occurred in $21 \%$ of the injured ankles. They occurred significantly more often in West Point grade III compared to IIB injuries (40\% vs. $12 \% p=0.0019)$. A multivariable analysis revealed that injury severity and the presence of a talar cartilage injury were significant negative predictors for return to sport-specific rehabilitation $(p<0.001 / p=0.023)$, return to team training $(p<0.001 / p=0.01)$ and return to match play $(p<0.001 / p=0.031)$. Unfortunately, concomitant intra-articular pathologies were only a secondary outcome parameter, and therefore, neither the severity of the chondral lesions, nor the subsequent treatment was reported. This lack of literature highlights the necessity for more detailed studies on intra-articular pathologies in acute isolated, unstable syndesmotic injury.

In the herein presented patient cohort, almost $50 \%$ of patients suffered intra-articular lesions. More importantly, $19 \%$ of patients required additional treatment because of intra-articular lesions. In contrast to the study by D'Hooghe et al. [8], neither the type of syndesmotic injury (bony vs. ligamentous; ns) nor the degree of ligamentous instability (West Point IIB vs. III; ns) had a significant influence on the occurrence of chondral lesions. This could be explained by the limited sample size. Still, the herein presented study highlights for the first time, the high frequency of intraarticular pathologies in detail for acute isolated, unstable syndesmotic injuries, reports on the subsequent treatments and compares their frequency to pre-operative MRI findings.

MRI is the current standard diagnostic tool to identify intra-articular lesions pre-operatively. In the current study, pre-operative MRI had a sensitivity/specificity of 100/79\% for chondral lesions and 50/93\% for loose bodies. The herein observed sensitivity compares favorably to previous studies with reported sensitivity rates ranging from 19 to $71 \%$ $[2,18,26,30]$. This high rate could be explained either by the rather small cohort or the experience of the senior radiologist reviewing the images especially for intra-articular pathologies. The sensitivity of $50 \%$ for loose bodies compared well to a previous study by O'Neill et al. [26]. The positive- $(0.5 / 0.5)$ and negative predictive values (1/0.93) for chondral lesions/loose bodies again compared well to the literature [18]. In our particular case, 6 of 17 patients (35\%) would have classified for additional arthroscopy based on the pre-operative MRIs (ICRS grad IV \pm loose bodies). Although MRI did detect all ICRS grade IV chondral lesions $(100 \%)$, one patient with an intraarticular body was missed (50\%). In three patients the MRI was false positive.
In accordance with the present literature, these findings underline the higher diagnostic accuracy for arthroscopy compared to MRI in detecting intra-articular lesions [24, 33]. Still, additional arthroscopy bears the risk for additional complications. In our cohort there was one instance of surgical site infection (4\%). A recent comprehensive literature review analyzed the complications following anterior and posterior ankle arthroscopy based on 55 studies [41], six of which included more than 100 patients $[1,9,16,29,40,42]$. These six studies reported a cumulative complication rate of $4.9 \%$ in 2676 patients. Lesions to the Superficial Peroneal Nerve occurred in $1.7 \%$, surgical site infections including sinus tract formation in $1.5 \%$, other nerve injuries in $1.3 \%$, deep vein thrombosis in $0.1 \%$, vascular injuries in $0.04 \%$, and other complications in $0.3 \%$ of patients. In summary, ankle arthroscopy appears to have a limited complication rate of about $5 \%$.

The key question for our clinical practice, therefore, is whether to base the indication for additional arthroscopy on pre-operative MRI, or to perform additional arthroscopy in all patients suffering an acute isolated, unstable syndesmotic? If the pre-operative MRI is chosen, there is a risk to miss a percentage of patients with intra-articular pathologies that would have necessitated treatment. On the other hand, treating all patients with additional arthroscopy might increase the total number of complications. We believe, that for now, the data available is not sufficient to answer this complex question comprehensively. This is predominantly because the influence of additional arthroscopy on the subjective, patient reported outcome is unknown for acute isolated, unstable syndesmotic injuries.

Following this study, our treatment strategy was adapted. For patients with high physical demands, additional arthroscopy is recommended, independent of the pre-operative MRI. In older patients with moderate level of activity, treatment recommendation are based on the preoperative MRI. This is done for several reason: first, micro-/nanofracturing has been shown to result in a superior outcome compared to debridement only for full-thickness cartilage lesions [10]; second, various studies on other pathologies, such as chronic ankle instability [23,39] or complex ankle fractures [34, 35], were able to demonstrate that arthroscopic assisted surgery with immediate treatment of intra-articular pathologies seems superior to conventional (non-arthroscopic) treatment; third, D'Hooghe et al. [8] were able to show, that talar cartilage lesions were a significant negative predictor for return to sports in this cohort. Therefore, one should not risk to miss any intra-articular pathology in highly active patients and rather accept the risk for a complication.

The study has several limitations, which should be discussed. First of all, the retrospective design. Although MRIs were only available in $74 \%$ of patients, the primary outcome parameter, i.e. intra-articular pathologies detected by 
arthroscopy, were generated from the surgical reports, which were available for all patients. Therefore, the risk of bias due to the retrospective data assessment could be considered as low. A further limitation might have been a selection bias. Only patients seen through by the Foot and Ankle Division were treated with additional arthroscopy. Although this can be rated as a pseudo-randomization, one could assume, that more active patients were referred to our outpatient clinic. Still, although the type of injury (ligamentous vs. bony) varied significantly between the two cohorts, none of the further assessed parameters did. Finally, the overall sample size of 27 is rather small, but the largest published so far.

\section{Conclusion}

Intra-articular pathologies occur in up to $50 \%$ of patients suffering an acute isolated, unstable syndesmotic injury. Nineteen percent of patients suffered ICRS grade IV lesions or had loose bodies that necessitated additional treatment. Whether the indication for additional arthroscopy should be based solely on pre-operative MRI remains unclear. For aged patients with a moderate level of activity, we base the indication for additional arthroscopy on the pre-operative MRI, due to its considerably high negative predictive value. In highly active patients, we routinely perform additional arthroscopy as we do not want to risk missing any intra-articular pathology and rather take the risk for a complication.

Acknowledgements Open Access funding provided by Projekt DEAL. We would like to thank Marissa Stagg, BSc for proofreading our manuscript.

Author contributions KR collected the data, helped to analyze the data, and wrote the paper; CB reviewed the MRIs and proof read the revised manuscript. JU structured the systematic review, helped in data interpretation and did final proof reading; TJV held substantial contributions in the conception and design of the work as well as final proof read of paper; WB held substantial contributions in the conception and data analysis as well as proof reading of the manuscript; HP conceived the original idea, conducted the data analysis and revised and finally proof read the manuscript; SFB conceived the original idea, was responsible for ethical approval and data bank registration as well as data bank analysis and paper preparation. All authors provided critical feedback and approved the final version to be published. The authors agree to be held accountable for the author's own contributions and ensure that questions related to the accuracy or integrity of any part of the work, even ones in which the author was not personally involved, are appropriately investigated and resolved.

Funding The authors received no specific funding for this work.

\section{Compliance with ethical standards}

Conflict of interest There is no potential conflict of interest of any of the authors. This manuscript has not been published elsewhere and is not under consideration by another journal. All authors agree with the submission to KSSTA

Ethical approval The study was approved by the local ethics committee (\#19-868)

Open Access This article is licensed under a Creative Commons Attribution 4.0 International License, which permits use, sharing, adaptation, distribution and reproduction in any medium or format, as long as you give appropriate credit to the original author(s) and the source, provide a link to the Creative Commons licence, and indicate if changes were made. The images or other third party material in this article are included in the article's Creative Commons licence, unless indicated otherwise in a credit line to the material. If material is not included in the article's Creative Commons licence and your intended use is not permitted by statutory regulation or exceeds the permitted use, you will need to obtain permission directly from the copyright holder. To view a copy of this licence, visit http://creativecommons.org/licenses/by/4.0/.

\section{References}

1. Ahn JH, Park D, Park YT, Park J, Kim Y-C (2019) What should we be careful of ankle arthroscopy? J Orthop Surg 27(3):2309499019862502

2. Bauer JS, Barr C, Henning TD, Malfair D, Ma CB, Steinbach L, Link TM (2008) Magnetic resonance imaging of the ankle at 3.0 Tesla and 1.5 Tesla in human cadaver specimens with artificially created lesions of cartilage and ligaments. Investig Radiol 43(9):604-611

3. Beumer A (2009) Chronic instability of the anterior syndesmosis of the ankle. Acta Orthop 78(Supp 327):2-40

4. Braunstein M, Baumbach SF, Urresti-Gundlach M, Borgmann L, Böcker W, Polzer H (2020) Arthroscopically assisted treatment of complex ankle fractures: intra-articular findings and 1-year follow-up. J Foot Ankle Surg 59(1):9-15

5. Brittberg M, Winalski CS (2003) Evaluation of cartilage injuries and repair. J Bone Jt Surg 85(A Suppl 2):58-69

6. Calder JD, Bamford R, Petrie A, McCollum GA (2016) Stable versus unstable grade II high ankle sprains: a prospective study predicting the need for surgical stabilization and time to return to sports. Arthroscopy 32(4):634-642

7. Choi WJ, Lee JW, Han SH, Kim BS, Lee SK (2008) Chronic lateral ankle instability: the effect of intra-articular lesions on clinical outcome. Am J Sports Med 36(11):2167-2172

8. D'Hooghe P, Grassi A, Alkhelaifi K, Calder J, Baltes TPA, Zaffagnini S, Ekstrand J (2019) Return to play after surgery for isolated unstable syndesmotic ankle injuries (West Point grade IIB and III) in 110 male professional football players: a retrospective cohort study. Br J Sports Med. https://doi.org/10.1136/bjsports-2018100298

9. Deng DF, Hamilton GA, Lee M, Rush S, Ford LA, Patel S (2012) Complications associated with foot and ankle arthroscopy. J Foot Ankle Surg 51(3):281-284

10. Duramaz A, Baca E (2018) Microfracture provides better clinical results than debridement in the treatment of acute talar osteochondral lesions using arthroscopic assisted fixation of acute ankle fractures. Knee Surg Sports Traumatol Arthrosc 26(10):3089-3095

11. D'Hooghe P, Alkhelaifi K, Abdelatif N, Kaux JF (2018) From "low" to 'high' athletic ankle sprains: a comprehensive review. Oper Tech Orthop 28:54-60 
12. Elias I, Zoga AC, Morrison WB, Besser MP, Schweitzer ME, Raikin SM (2007) Osteochondral lesions of the talus: localization and morphologic data from 424 patients using a novel anatomical grid scheme. Foot Ankle Int 28(2):154-161

13. Espinosa N, Smerek JP, Myerson MS (2006) Acute and chronic syndesmosis injuries: pathomechanisms, diagnosis and management. Foot Ankle Clin 11(3):639-657

14. Feldman MD (2016) Editorial commentary: does early arthroscopy of subtle instability in high ankle sprains hasten return to play in elite athletes? Arthroscopy 32(4):643-644

15. Ferkel RD, Chams RN (2016) Chronic lateral instability: arthroscopic findings and long-term results. Foot Ankle Int 28(1):24-31

16. Ferkel RD, Heath DD, Guhl JF (1996) Neurological complications of ankle arthroscopy. Arthroscopy 12(2):200-208

17. Frick H (1986) Diagnosis, therapy and results of acute instability of the syndesmosis of the upper ankle joint (isolated anterior rupture of the syndesmosis). Der Orthopade 15(6):423-426

18. Gatlin CC, Matheny LM, Ho CP, Johnson NS, Clanton TO (2015) Diagnostic accuracy of 3.0 Tesla magnetic resonance imaging for the detection of articular cartilage lesions of the talus. Foot Ankle Int 36(3):288-292

19. Gerber JP, Williams GN, Scoville CR, Arciero RA, Taylor DC (1998) Persistent disability associated with ankle sprains: a prospective examination of an athletic population. Foot Ankle Int 19(10):653-660

20. Hintermann B, Regazzoni P, Lampert C, Stutz G, Gächter A (2000) Arthroscopic findings in acute fractures of the ankle. J Bone Jt Surg Br 82(3):345-351

21. Hopkinson WJ, St Pierre P, Ryan JB, Wheeler JH (1990) Syndesmosis sprains of the ankle. Foot Ankle 10(6):325-330

22. Hua Y, Chen S, Li Y, Chen J, Li H (2010) Combination of modified Broström procedure with ankle arthroscopy for chronic ankle instability accompanied by intra-articular symptoms. Arthroscopy 26(4):524-528

23. Karlsson J, Bergsten T, Lansinger O, Peterson L (1988) Reconstruction of the lateral ligaments of the ankle for chronic lateral instability. J Bone Jt Surg Am 70(4):581-588

24. Krähenbühl N, Weinberg MW, Mills MK, Hintermann B, Barg A (2018) Assessment and treatment of chronic syndesmotic instability. Fuss Sprunggelenk 16:264-274

25. Mulcahey MK, Bernhardson AS, Murphy CP, Chang A, Zajac T, Sanchez G, Sanchez A, Whalen JM, Price MD, Clanton TO, Provencher MT (2018) The epidemiology of ankle injuries identified at the National Football League Combine, 2009-2015. Orthop J Sports Med 6(7):2325967118786227. https://doi. org/10.1177/2325967118786227

26. O'Neill PJ, Van Aman SE, Guyton GP (2010) Is MRI adequate to detect lesions in patients with ankle instability? Clin Orthop Relat Res 468(4): 1115-1119

27. Porter DA (2009) Evaluation and treatment of ankle syndesmosis injuries. Instr Course Lect 58:575-581

28. Randell M, Marsland D, Ballard E, Forster B, Lutz M (2019) MRI for high ankle sprains with an unstable syndesmosis: posterior malleolus bone oedema is common and time to scan matters. Knee Surg Sports Traumatol Arthrosc 27(9):2890-2897
29. Rasmussen S, Hjorth Jensen C (2002) Arthroscopic treatment of impingement of the ankle reduces pain and enhances function. Scand J Med Sci Sports 12(2):69-72

30. Rolf CG, Barclay C, Riyami M, George J (2006) The importance of early arthroscopy in athletes with painful cartilage lesions of the ankle: a prospective study of 61 consecutive cases. J Orthop Surg Res. 1:4

31. Ræder BW, Figved W, Madsen JE, Frihagen F, Jacobsen SB, Andersen MR (2020) Better outcome for suture button compared with single syndesmotic screw for syndesmosis injury: five-year results of a randomized controlled trial. Bone $\mathrm{Jt} \mathbf{J}$ 102-B(2):212-219

32. Scranton PE, McDermott JE, Rogers JV (2000) The relationship between chronic ankle instability and variations in mortise anatomy and impingement spurs. Foot Ankle Int 21(8):657-664

33. Staats K, Sabeti-Aschraf M, Apprich S, Platzgummer H, Puchner SE, Holinka J, Windhager R, Schuh R (2017) Preoperative MRI is helpful but not sufficient to detect associated lesions in patients with chronic ankle instability. Knee Surg Sports Traumatol Arthrosc 26(7):2103-2109

34. Takao M, Uchio Y, Naito K, Fukazawa I, Kakimaru T, Ochi M (2004) Diagnosis and treatment of combined intra-articular disorders in acute distal fibular fractures. J Trauma 57(6):1303-1307

35. Turhan E, Doral MN, Demirel M, Atay AO, Bozkurt M, Bilge O, Huri G, Atesok K, Kaya D (2013) Arthroscopy-assisted reduction versus open reduction in the fixation of medial malleolar fractures. Eur J Orthop Surg Traumatol 23(8):953-959

36. Valkering KP, Vergroesen DA, Nolte PA (2012) Isolated syndesmosis ankle injury. Orthopedics 35(e):1705-1710

37. van Dijk CN, Longo UG, Loppini M, Florio P, Maltese L, Ciuffreda M, Denaro V (2016) Classification and diagnosis of acute isolated syndesmotic injuries: ESSKA-AFAS consensus and guidelines. Knee Surg Sports Traumatol Arthrosc 24(4):1200-1216

38. Vopat ML, Vopat BG, Lubberts B, DiGiovanni CW (2017) Current trends in the diagnosis and management of syndesmotic injury. Curr Rev Musculoskelet Med 10(1):94-103

39. Yeo ED, Lee K-T, Sung I-H, Lee SG, Lee YK (2016) Comparison of all-inside arthroscopic and open techniques for the modified Broström procedure for ankle instability. Foot Ankle Int 37(10):1037-1045

40. Young BH, Flanigan RM, DiGiovanni BF (2011) Complications of ankle arthroscopy utilizing a contemporary noninvasive distraction technique. J Bone Jt Surg Am 93(10):963-968

41. Zekry M, Shahban SA, El Gamal T, Platt S (2019) A literature review of the complications following anterior and posterior ankle arthroscopy. Foot Ankle Surg 25(5):553-558

42. Zengerink M, van Dijk CN (2012) Complications in ankle arthroscopy. Knee Surg Sports Traumatol Arthrosc 20(8):1420-1431

Publisher's Note Springer Nature remains neutral with regard to jurisdictional claims in published maps and institutional affiliations. 